\title{
Roadmap für eine nachhaltige Informationstechnik
}

\author{
Nachhaltige Entwicklung und Informations- und Wissensgesellschaft sind die \\ zwei großen Leitbilder, die heute die Zukunftsdebatten bestimmen. Es kommt \\ darauf an, diese beiden Leitbilder zu verknïpfen. Der Bundestag hat die Grund- \\ lagen dafür gelegt, dass eine in Zusammenarbeit von Unternehmen und Wissen- \\ schaft entwickelte Roadmap den notwendigen Orientierungsrahmen bietet.
}

\begin{abstract}
A Von Ulla Burchardt uch wenn die New Economy nach überspannten Erwartungen und einem irrationalen Höhenflug an den Börsen nun wieder auf dem harten Boden der Tatsachen angekommen ist, besteht doch kein Zweifel: Wissen und Information sind schon heute Wirtschaftsfaktoren ersten Ranges. Die neuen Informationstechniken haben unsere Art zu leben, zu arbeiten, zu wirtschaften und zu kommunizieren grundlegend verändert und werden dies in Zukunft weiter tun.

Bislang noch weitgehend unvermittelt zur Leitidee der Wissensgesellschaft, gewinnt das andere große Leitbild, die Nachhaltigkeit, immer mehr an öffentlicher Aufmerksamkeit. Zunehmend wächst das Bewusstsein darüber, dass der bisherige Wachstumspfad, auf dem der Wohlstand der reichen Länder erreicht wurde, an ökologische und soziale Grenzen stößt und auf Dauer nicht tragfähig ist. Das Leitbild Nachhaltigkeit setzt dem eine positive Vision, ein neues Fortschrittsmodell entgegen. Für die wohlhabenden Staaten lässt es sich auf eine einfache Formel bringen: mehr Wohlstand und mehr Lebensqualität durch weniger Energie- und Ressourcenverbrauch, weniger Emissionen, Schadstoffe und Abfälle.
\end{abstract}

\section{- Verknüpfung beider Leitbilder}

Es gilt die digitale Kluft nicht weiter wachsen zu lassen, sondern zu schließen und die bedeutendste Wachstumsbranche der Gegenwart und Zukunft auf eine nachhaltige Wirtschaftsweise einzustellen. Nur so können die technologischen Chancen optimalen gesellschaftlichen Nutzen entfalten.

Der Übergang von der Industrie- zur Wissensgesellschaft bildet eine gute Voraussetzung, um wirtschaftliche Entwicklung und Ressourcenverbrauch voneinander zu entkoppeln - Stichwort: Dematerialisierung. Intelligente informationstech- nische Lösungen bieten zum Beispiel erhebliche Potenziale zur Reduzierung von Energieverbrauch und Schadstoffemissionen durch IuK-gestïtztes Gebäudemanagement oder bei der Verkehrslenkung und -vermeidung.

Diesen positiven Potenzialen stehen allerdings auch negative Trends entgegen. Das betrifft zum einen die Hardware, die von der Herstellung bis zur Entsorgung eine Fülle von Umweltproblemen aufwirft. Zum anderen tragen die IuK-Techniken zur Entgrenzung und Beschleunigung von Marktprozessen bei. Die erreichten Effizienzsteigerungen werden dadurch wieder aufgefressen die bekannte Rebound-Falle.

\section{Innovationsförderung mit Weitblick}

In der 13. Wahlperiode des Bundestages hat die Enquete-Kommission „Schutz des Menschen und der Umwelt" den Versuch unternommen, das Leitbild Nachhaltigkeit in ein Handlungskonzept zu übersetzen (1). Die Informations- und Kommunikationstechnik wurde als ein konkretes Handlungsfeld ausgewählt. Die beiden besonderen Herausforderungen bei der Entwicklung von Handlungsstrategien waren und sind die Internationalität der Branche und die extrem kurzen Innovationszyklen. Reaktive gesetzliche Maßnahmen im nationalen Rahmen - so unverzichtbar sie sind - werden alleine nicht ausreichen, um eine Trendwende in Richtung Nachhaltigkeit in der Informationstechnik auszulösen.

Gestïtzt auf wissenschaftliche Expertise des Instituts für Zukunftsstudien und Technologiebewertung (IZT), Berlin, hat die Enquete deshalb als ein wichtiges Instrument die Erarbeitung eines branchenweiten Orientierungsrahmens, einer sogenannten Roadmap, vorgeschlagen. Dabei geht es darum, dass sich die Unternehmen und ihre Verbände in Kooperation mit der Wissenschaft selbst über vordringlichen Handlungsbedarf verständigen und langfristige Innovationspfade für eine sozial- und umweltverträgliche Gestaltung des Fortschritts in der Informations- und Kommunikationstechnik aufzeigen. Im Endeffekt sollte dieser Prozess möglichst in verbindliche Selbstverpflichtungen der Beteiligten münden.

Die Vorteile dieses Instruments liegen auf der Hand. Es ist flexibel, lässt sich relativ rasch an neue technologische Trends anpassen und kann dazu beitragen, dass bereits in der Entwicklungsphase die Entstehung von Problemen vermieden wird, die ansonsten später nur schwer zu lösen sein werden. Bei der Elektroschrottproblematik ist derzeit zu erleben, zu welchen Schwierigkeiten es führt, wenn nicht frühzeitig die Weichen richtig gestellt werden. Hinzu kommt, dass das Instrument den Unternehmen Richtungssicherheit gibt und sie aktiv in die Verantwortung nimmt. Es kann ordnungsrechtliche Maßnahmen nicht ersetzen. Sollte die Branche das Instrument allerdings aktiv nutzen, könnten sich gesetzliche Regelungen im Einzelfall durchaus erübrigen.

\section{Bewährte Methode}

Die Methode des Roadmapping ist ein bewährtes Instrument der strategischen Unternehmensplanung und Innovationsförderung. Und auch im Hinblick auf ökologisch orientierte Ansätze des Roadmapping gibt es bereits vorzeigbare Beispiele, wie die Initiative der Microelectronics and Computer Technology Corporation, die bereits Anfang der 90er-Jahre erstmals eine „Electronic Industry Environmental Roadmap" entwickelt hat. Sie wurde bis 1996 mehrfach aktualisiert. In Japan wurde der Ansatz in jüngerer Zeit vom Ministerium für Handel und Industrie aufgegriffen. Und auch in Deutschland ist diese Methodik durchaus geläufig, wenn auch für die Entwicklung ökologischer Langfriststrategien noch nicht erprobt. Allerdings gibt es eine Reihe von Initiativen, die auf eine ökologische Optimierung der Informations- und Kommunikationstechnik zielen. Man denke an das vom Bundesforschungsministerium (BMBF) geförderte Projekt „Grüner Fernseher", das EUREKA-Projekt CARE VISION 2000 oder das Konzept der AG CYCLE.

\section{Impulse aus dem Parlament}

Ein Manko dieser Aktivitäten bestand bislang jedoch darin, das sie eher fragmentarischen Charakter hatten und untereinander wenig vernetzt waren.

Zu Beginn dieser Wahlperiode haben daher die Koalitionsfraktionen auf Initiative der SPD den 
Antrag ,Strategie für eine nachhaltige Informationstechnik" in den Bundestag eingebracht und so den entscheidenden Impuls für die Umsetzung der Enquete-Empfehlung gegeben (2). Die Bundesregierung wurde aufgefordert, die Initiative für die Entwicklung einer Roadmap zu ergreifen.

Bei einem Workshop der SPD-Fraktion mit Vertretern aus Unternehmen, Verbänden, Wissenschaft und den zuständigen Bundesministerien sind erste Leitlinien für das mögliche Vorgehen entwickelt worden. Beteiligt waren mit dem Branchenverband BITKOM, den Unternehmen IBM, Siemens und Infineon einige wichtige Größen der IuK-Branche. Ergebnis dieses ersten Brainstormings war ein abgestimmtes Eckpunktepapier. Die Teilnehmer gingen ein erstes „Commitment“ ein, in dem sie sich grundsätzlich offen und interessiert zeigten, aktiv im Rahmen einer solchen Innovationspartnerschaft zwischen Staat, Industrie und Wissenschaft mitzuarbeiten.

Unter den Beteiligten war unstrittig, dass ein Orientierungsrahmen für Nachhaltigkeit in der Informationstechnik im wohlverstandenen Eigeninteresse der Unternehmen liegt. Er gibt ökologische Richtungssicherheit, stimuliert Innovationen, sorgt für mittel- bis langfristige Planungssicherheit und fördert Transparenz und Effizienz durch Bündelung bereits vorhandener Maßnahmen und Initiativen. Dieser Konsens ist eine Grundvoraussetzung für die erfolgreiche Anwendung des Instruments, auf das Unternehmen sich nur dann einlassen werden, wenn sie sich davon einen Ertrag versprechen.

Struktur und wesentliche Bausteine einer Roadmap wurden skizziert. Als Kernelemente kristallisierten sich Status-Analysen und Problemdefinitionen, Zielvorgaben und die Entwicklung von Szenarien, eine Auswahl prioritärer Handlungsfelder, eine klare Aufgabenverteilung unter den Akteuren und Methoden des Monitorings heraus.

\section{Das Projekt NIK}

Damit waren erste Pflöcke gesetzt und ein grober Rahmen abgesteckt. Das BMBF hat die Empfehlungen aufgegriffen und zur Grundlage der weiteren Planungen gemacht. Die Konzeption des Vorhabens wurde an den Projektträger DLR übertragen. Das Projekt „Nachhaltigkeit in der Informations- und Kommunikationstechnik" (NIK) wurde aus der Taufe gehoben.

Seit etwa Mitte letzten Jahres ist ein Projektteam bestehend aus dem Fraunhofer-Institut für Zuverlässigkeit und Mikrointegration, dem IZT und dem Beratungsbüro für Umwelt- und Unternehmensent- wicklung damit beauftragt, den Dialogprozess für eine Roadmap der IuK-Branche zu organisieren, zu moderieren und wissenschaftlich zu begleiten.

Als erster Schritt wurde ein Arbeitskreis eingerichtet, in dem Industrie, Forschung und Politik vertreten sind. Dieser Arbeitskreis ist Lenkungsgremium und Keimzelle des Dialogprozesses, der offiziell mit einer Auftaktveranstaltung im Rahmen der Messe Productronica in München im November 2001 begann. Ziel dieses ersten Expertenforums war es vor allem, weitere Unternehmen für die Teilnahme zu gewinnen, denn eine breite Beteiligung ist unverzichtbar für die Aussagekraft und Verbindlichkeit der Roadmap.

Zwischenzeitlich zeichnen sich erste Konturen der inhaltlichen Schwerpunkte des Projekts ab. Aufbauend auf einer Trendanalyse und einer Unternehmensbefragung zum Status quo und den wichtigsten Handlungsfeldern wurden im Arbeitskreis drei Fokusthemen identifiziert:

- nachhaltige Entwicklung in der mobilen Kommunikation,

- Bildschirmrecycling und innovative Displaytechnologien und

- öffentliche Beschaffung von und mit IuK-Technik.

Sie decken wichtige Felder der technologischen Entwicklung und des Marktgeschehens ab und sind mit unterschiedlichen strategischen Handlungsansätzen verknüpft, denen aus Unternehmenssicht besondere Bedeutung zukommt.

So wird der Schwerpunkt im Feld der mobilen Kommunikation unter dem Stichwort „Design for Environment" auf einer umweltgerechten Produktentwicklung im Zuge der Umstellung der Infrastruktur und der Endgeräte auf die dritte Mobilfunkgeneration (UMTS) liegen. Das Fokusthema Displays wird vorrangig um Fragen der Ressourceneffizienz, der Produktnutzung und des Recyclings kreisen. Und im Feld öffentliche Beschaffung wird es um Strategien gehen, die durch unternehmerische und politische Maßnahmen die Nachfragemacht der öffentlichen Hand für eine nachhaltige Entwicklung stärker nutzbar machen.

Für alle drei Felder wurden eigene Fokusgruppen mit Vertretern aus Unternehmen und Wissenschaft gebildet, deren Aufgabe es ist, erwartbare Trends zu identifizieren, mögliche $\mathrm{Zu}$ kunftsvisionen $\mathrm{zu}$ entwickeln und beides zusammenzuführen. Der Weg vom Trend zur Vision wird auf Machbarkeit zu prüfen und in einem Schritteprozess abzubilden sein. Für alle drei Themen werden Roadmaps entwickelt, deren erste Entwürfe im Rahmen eines zweiten Expertenforums diesen Mai in Berlin vorgestellt wurden.

\section{- Viel versprechendes Experiment}

Noch ist es zu früh, um einschätzen zu können, ob das Projekt NIK nachhaltige Erfolge bringen wird. Das Vorhaben ist ein Experiment. Aber schon jetzt lässt sich feststellen: Es ist ein spannender Prozess in Gang gesetzt worden, weite Teile der Branche sind in den Dialog eingebunden, ein Netzwerk entsteht und die Arbeit macht schnelle Fortschritte. Die Zeichen stehen gut, dass aus dem staatlich geförderten Vorhaben ein selbsttragender Prozess werden kann.

Allerdings wird es notwendig sein, dem Projekt einen festen Platz innerhalb bestehender Unternehmenskooperationen, -verbünde und laufender Initiativen einzuräumen. Die Einbeziehung von NIK in die Initiative D21 ist wünschenswert. Es macht durchaus Sinn, ein derartiges Projekt auf nationaler Ebene anzustoßen - das jüngste Gutachten des Sachverständigenrates für Umweltfragen hat noch einmal unterstrichen, dass eine Vorreiterrolle bei der sozial-ökologischen Modernisierung gerade auch unter ökonomischen Gesichtspunkten Vorteile bringen kann (3). Auf Sicht allerdings sollte in einer Branche wie der Informationswirtschaft, die keine nationalen Grenzen kennt, aus einem nationalen ein europäisches Projekt werden.

\section{Anmerkungen}

(1) Enquete-Kommission „Schutz des Menschen und der Umwelt - Ziele und Rahmenbedingungen einer nachhaltig zukunftsverträglichen Entwicklung": Konzept Nachhaltigkeit Vom Leitbild zur Umsetzung. Abschlussbericht. BundestagsDrucksache 13/11200.

(2) Antrag von SPD und Bündnis 90/ Die Grünen vom 14.12.1999, Bundestags-Drucksache 14/2390; Beschlussempfehlung und Bericht des Ausschusses für Bildung, Forschung und Technikfolgenabschätzung vom 25.02.2000, Drucksache 14/2814.

(3) Rat von Sachverständigen für Umwelffragen: Umweltgutachten 2002. Für eine neve Vorreiterrolle. Berlin 2002. Kurzfassung im Internet unter http://www.umweltrat.de/gutach02.htm

\section{Die Autorin}

Ulla Burchardt ist stellvertretende Vorsitzende des Ausschusses für Bildung, Forschung und Technikfolgenabschätzung des Deutschen Bundestages und Sprecherin der SPD-Fraktion für Nachhaltige Entwicklung. Kontakt: Deutscher Bundestag, 11011 Berlin. Tel. 030/ 227-75856, Fax -76303, E-mail: ulla.burchardt@bundestag.de 
(c) 20I0 Authors; licensee IÖW and oekom verlag. This is an article distributed under the terms of the Creative Commons Attribution Non-Commercial No Derivates License (http://creativecommons.org/licenses/by-nc-nd/3.o/), which permits unrestricted use, distribution, and reproduction in any medium, provided the original work is properly cited. 